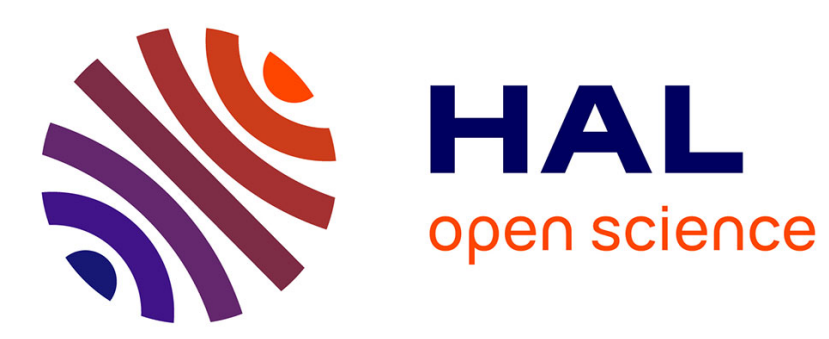

\title{
The Proportional Colouring Problem: Optimizing Buffers in Radio Mesh Networks
}

Florian Huc, Claudia Linhares Sales, Hervé Rivano

\section{To cite this version:}

Florian Huc, Claudia Linhares Sales, Hervé Rivano. The Proportional Colouring Problem: Optimizing Buffers in Radio Mesh Networks. The IV Latin-American Algorithms, Graphs, and Optimization Symposium (LAGOS 07), Feb 2008, Puerto Varas, Chile. pp.141-146. hal-00429822

\section{HAL Id: hal-00429822 \\ https://hal.science/hal-00429822}

Submitted on 4 Nov 2009

HAL is a multi-disciplinary open access archive for the deposit and dissemination of scientific research documents, whether they are published or not. The documents may come from teaching and research institutions in France or abroad, or from public or private research centers.
L'archive ouverte pluridisciplinaire HAL, est destinée au dépôt et à la diffusion de documents scientifiques de niveau recherche, publiés ou non, émanant des établissements d'enseignement et de recherche français ou étrangers, des laboratoires publics ou privés. 


\title{
The Proportional Colouring Problem: Optimizing Buffers in Wireless Mesh Networks
}

\author{
Florian Huc ${ }^{1}$ \\ I3S (CNRS-UNSA), INRIA \\ Sophia Antipolis, France \\ Cláudia Linhares-Sales ${ }^{1}$ \\ Universidade Federal do Ceará, Brazil \\ MASCOTTE Project/INRIA, France \\ Hervé Rivano ${ }^{1}$ \\ I3S (CNRS-UNSA), INRIA \\ Sophia Antipolis, France
}

\begin{abstract}
In this paper, we consider a new edge colouring problem motivated by wireless mesh networks optimization: the proportional edge colouring problem. Given a graph $G$ with positive weights associated to its edges, we want to find a proper edge colouring which assigns to each edge at least a proportion (given by its weight) of all the colours. If such colouring exists, we want to find one using the minimum number of colours. We proved that deciding if a weighted graph admits a proportional edge colouring is polynomial while determining its proportional edge chromatic number is NP-hard. We also give a lower and an upper bound that can be polynomially computed. We finally characterize some graphs and weighted graphs for which we can determine the proportional edge chromatic number.
\end{abstract}

Keywords: edge colouring, proportional colouring, wireless mesh network. 


\section{Introduction}

Given a weighted graph $(G, w)$ where $w$ is a weight function from $E(G)$ to $\mathbb{R}^{+}$, several distinct colouring problems of $G$ have been defined. In [2], one wants to colour the vertices of $G$ while minimizing the sum of the weights of the edges whose extremities receive the same colour. In [5], one wants to colour the vertices of $G$ so that for each edge $u v,|c(v)-c(u)| \geq w(u v)$, where $c(u)$ and $c(v)$ are the colours assigned to $u$ and $v$. In this paper, we consider a proper edge colouring which assigns to each edge $e$ at least a fraction (given by the weight of $e$ ) of all the colours used. By "proper", we mean that, for any two adjacent edges, the sets of assigned colours have an empty intersection. If such colouring exists, we want to find one using the minimum number of colours, number which we call proportional edge chromatic number.

In next section, we introduce the notations we will use throughout this paper and recall some results about edge colouring. Then we will present the telecommunication problem which is modelled by the proportional edge colouring problem, together with some complexity results and bounds for the proportional edge chromatic number. Finally, we characterize a class of graphs and a class of weighted graphs whose proportional edge chromatic number can be computed in polynomial time.

\section{Preliminaries}

Throughout the paper, $(G, w)$ denotes a weighted simple graph where $w$ is a positive function called weight function defined on the edges of $G, w: E(G) \rightarrow$ $[0,1]$. We denote by $\Delta(G)$ the maximum degree of $G$.

The classical edge colouring problem is to determine the edge chromatic number of a simple graph $G, \chi^{\prime}(G)$, that is, the minimum integer $k$ such that $G$ admits a proper edge colouring using $k$ colours. In 1964, Vizing proved that $\chi^{\prime}(G)$ is at most $\Delta+1[7]$. Since it is at least $\Delta$, we can classify every graph: a graph is in Class 1 if its edge chromatic number is $\Delta$ and in Class 2 otherwise. Surprisingly, deciding if a graph is Class 1 or 2 is hard [3], even for cubic graphs [4]. For the upper bound presented in this work, we recall a possible definition of the fractional edge chromatic number: $\chi^{\prime *}=\min _{k \geq 1} \frac{\chi^{\prime}\left(G^{k}\right)}{k}$, where $G^{k}$ is the graph obtained from $G$ by replacing each edge by $k$ parallel edges. Also recall that this parameter can be determined in polynomial time [6].

${ }^{1}$ C. Linhares-Sales is partially supported by CNPq/Brazil. F. Huc is supported by CNRS and région PACA. This work has been partially funded by European project IST/FET AEOLUS and ANR-JC OSERA. Email: Florian.Huc, Claudia.Linhares-Sales, Herve.Rivano@sophia.inria.fr 
Since we only consider proportional edge colouring, we will omit to precise edge in the remainder of the paper.

\section{Proportional colouring}

The proportional colouring problem is motivated by the following telecommunication problem. We consider a slotted time division multiplexing wireless mesh network connecting routers through directional antennas. We denote by call two antennas communicating together. The network topology defines a graph $G=(V, E)$ whose vertices are the routers and the edges are the achievable calls. For the sake of radio interferences and near-far effect, each router can be involved in at most one call at a time. Therefore, a set of simultaneously achievable calls is a matching of $G$. More precisely, given a set of calls, the classical timeslot assignment problem consists in decomposing this set into a minimum number of subsets of simultaneously achievable calls, which is equivalent to the proper edge colouring problem [1]. Remark that without the assumption of directional antennas, this problem would be modelled by the induced matching problem.

The proportional colouring problem arises when we consider Constant Bit Rates (CBR) requests. We are given a set of communication requests, each request being a source-destination path in the network, and a bit rate. Sending this amount of data on the paths induces that each call has to be periodically activated in a given proportion of the time. This is modelled by a weight function $w: E(G) \rightarrow[0,1]$.

The problem is now, if possible, to find a periodical schedule of the calls satisfying the CBR requests, that is, such that to each call a number of timeslots proportional to its weight is assigned to. Besides, the length of the period is proportional to the size of the buffer needed at each router, which is an important parameter of the cost of the network.

We therefore want a proper edge colouring of $G$ that satisfies the proportions given by the weights of the edges, with the minimum number of colours. This is the proportional colouring problem formally defined as follows.

Definition 3.1 [proportional colouring] Given a weighted graph $(G, w)$, a proportional colouring of $(G, w)$ is a function $\mathcal{C}: E \rightarrow \mathcal{P}(\{1, \ldots, c\})$ such that for all $e \in E$, we have: $|\mathcal{C}(e)| \geq c \cdot w(e)$ and for all adjacent edges $e, f$, $\mathcal{C}(e) \cap \mathcal{C}(f)=\emptyset$. We call the proportional chromatic number of $G, \chi_{\pi}^{\prime}(G, w)$, the minimum number of colours $c$ for which a proportional colouring of $G$ exists. If it does not exist then $\chi_{\pi}^{\prime}(G, w)=\infty$.

Definition 3.2 [m-graph] Given a weighted graph $(G, w)$ and an integer $m$, 


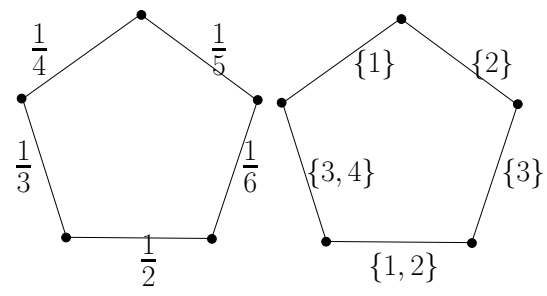

Fig. 1. A weighted $C_{5}$ and a proportional colouring using 4 colours

the $m$-graph $G_{m}$ is constructed on vertex set $V(G)$ as follows: given an edge $e=(u v) \in E(G)$, we put $\lceil m w(e)\rceil$ multiple edges $u v$ in $E\left(G_{m}\right)$.

Remark 3.3 Notice that given a weighted graph $(G, w)$ and an integer $m$, if a colouring using $\mathrm{km}$ colours (where $k$ is a constant) which gives at least $k$ colours to all the edges of $G_{m}$ exists, then this colouring can be easily transformed into a proportional colouring of $(G, w)$.

Definition 3.4 $[\operatorname{mcd}(\mathrm{w})]$ Given a weighted $\operatorname{graph}(G, w)$ with $w$ taking value in $\mathbb{Q}$, we set $\operatorname{mcd}(w)$ as the minimum common denominator of all the values taken by $w$.

Figure 1 is an example of a weighted graph and its proportional colouring. The proportional colouring problem is divided into two subproblems. The first one consists in proving that there exists an integer $c$ such that a $c$-proportional colouring of $(G, w)$ exists. The second one is to determine the proportional chromatic number of $(G, w)$. We start by giving simple facts which we will use to prove Theorem 3.6.

Fact 3.5 Let $(G, w)$ be a weighted graph.

- If there is a vertex $u$ of $G$ with $\sum_{u v \in E(G)} w(u v)>1$ then $\chi_{\pi}^{\prime}(G, w)=\infty$.

- If for all $u v \in E(G), w(u v) \leq 1 /(\Delta+1)$ then $\chi_{\pi}^{\prime}(G, w) \leq \Delta+1$.

- Similarly, if for all uv $\in E(G), w(u v) \leq 1 / \chi^{\prime *}\left(G_{m c d(w)}\right)$ then $\chi_{\pi}^{\prime}(G, w) \leq q$ where $q$ is the numerator of $\chi^{*}\left(G_{m c d(w)}\right)$.

Theorem 3.6 Let $(G, w)$ be a weighted graph with $w$ taking value in $\mathbb{Q}$.

i) Determining if there exists a proportional colouring for $(G, w)$ is polynomial.

ii) Determining the proportional chromatic number of $(G, w)$ is NP-hard.

\section{Proof.}

i) Let $(G, w)$ be any weighted graph with $w: E(G) \rightarrow[0,1] \cap \mathbb{Q}$. Let $m c d:=$ $\operatorname{mcd}(w)$. In the $m c d$-graph, we have $\chi^{\prime *}\left(G_{m c d}\right) \leq \operatorname{mcd} \Leftrightarrow \chi_{\pi}^{\prime}(G, w)<\infty$. 
Indeed, suppose $\chi_{\pi}^{\prime}(G, w)=k<\infty$. Then, there exists a proportional colouring using $k$ colours. If we repeat this colouring mcd times, we still have a proportional colouring, even if it is not minimal. This colouring gives a proper edge colouring of $G_{m c d}$ using $k \cdot \operatorname{mcd}$ colours, in which each edge receives at least $k$ colours. Therefore, $\chi^{* *}\left(G_{m c d}\right) \leq \frac{k \cdot m c d}{k}$. On the other hand, suppose that $\chi^{*}\left(G_{m c d}\right)=\frac{q}{p}$ and consider this optimal fractional edge colouring of $G_{m c d}$. This colouring can be extended to an edge colouring of $G$ that uses $q$ colours and assigns $p \cdot m c d \cdot w(e)$ to each edge $e$. This colouring is proportional, since, by assumption, $\mathrm{mcd} \cdot w(e) \cdot p \geq q \cdot w(e)$. Hence $\chi_{\pi}^{\prime}(G, w)=k \leq q<\infty$.

ii) Given a graph $G$ of maximum degree $\Delta$, we set the weights of its edges to $\frac{1}{\Delta+1}$. Since $G$ is $\Delta+1$ colourable, $G$ is proportionally $\Delta+1$ colourable. Now, observe that if $(G, w)$ admits a proper edge colouring with $\Delta$ colours, then it admits a proportional colouring with $\Delta$ colours. Since determining if $G$ is $\Delta$ or $\Delta+1$ colourable is NP-hard [3], it is NP-hard to determine the proportional chromatic number of an instance $(G, w)$.

In despite of the difficulty of computing the proportional chromatic number of a weighted graph $(G, w)$, we can deduce polynomially computable lower and upper bounds. Clearly, for a weighted graph $(G, w), \chi_{\pi}^{\prime}(G, w) \geq \Delta(G)$. Theorem 3.7 improves this lower bound.

Theorem 3.7 (Lower bound) Let $m$ be the minimum integer satisfying for all $u \in V, \sum_{v \text { st } u v \in E}\lceil m w(u v)\rceil \leq m$. If no $m$ satisfies all the above equations, then no proportional colouring exists. Otherwise, if there is a solution $m$ and that $(G, w)$ admits a proportional colouring, $\chi_{\pi}^{\prime}(G, w)$ is at least $m$.

Theorem 3.8 (Upper bound) Let $(G, w)$ be a weighted graph such that $w$ takes value in $\mathbb{Q}$. If $G, w)$ admits a proportional colouring, then there is a proportional colouring of $(G, w)$ using $q$ colours, where $q$ is the numerator of $\chi^{\prime *}\left(G_{m c d(w)}\right)$.

The proof of Theorem 3.8 follows from item (i) of Theorem 3.6. In general, given a weighted graph $(G, w)$, the mcd of the values taken by $w$ is not an upper bound. Indeed, consider $\left(P, \frac{\mathbf{1}}{\mathbf{3}}\right)$ : the Petersen graph $P$ with the constant weight function equal to $\frac{1}{3}$. Since $\chi^{\prime *}(P)=3$, we have $\chi_{\pi}^{\prime}\left(P, \frac{\mathbf{1}}{\mathbf{3}}\right)<\infty$. However $\chi_{\pi}^{\prime}\left(P, \frac{\mathbf{1}}{\mathbf{3}}\right)>3=\operatorname{mcd}\left(\frac{\mathbf{1}}{\mathbf{3}}\right)$, since $P$ is not 3 -colourable.

The proportional chromatic number may also be different from the upper bound given by Theorem 3.8, because an edge can receive proportionally more colours than it required. This is illustrated by Figure 1. Indeed, in this example, we have $\chi^{\prime *}\left(C_{5} \operatorname{mcd}(w)\right) \geq 50>4=\chi_{\pi}^{\prime}\left(C_{5}, w\right)$. 
One can also ask for which classes of graphs the proportional colouring problem can be polynomially solved. The next theorems announce two positive results, the first one giving a condition to the lower bound be reached.

Theorem 3.9 Let $(G, w)$ be a weighted bipartite graph. If there is a solution $m$ to the set of equations given by Theorem 3.7, then $(G, w)$ admits a proportional colouring using $m$ colours. In fact, for any weighted graph $(G, w)$, $\chi_{\pi}^{\prime}(G, w)=m \Leftrightarrow \chi^{\prime}\left(G_{m}\right)=m$.

Theorem 3.10 Let $(G, w)$ be a weighted graph with $w$ taking values in $\mathbb{Q}$. Let $e$ be an edge with an end vertex $v$ such that $\sum_{u v \in E} w(u v)=1$. Then, the denominator of $w(e)$ divides $\chi_{\pi}^{\prime}(G, w)$. In particular, if the proportional chromatic number of $(G, w)$ is finite and every edge e has an end vertex $v$ with $\sum_{u v \in E} w(u v)=1$, then $\chi_{\pi}^{\prime}(G, w)=\operatorname{mcd}(w)$.

Motivated by the applications modelled by the proportional colouring problem and its hardness, we pose the following general open questions: find approximation algorithms for the classes of graphs which usually occur in telecommunication, as circular-arc graphs and triangular lattices, and determine other classes of graphs (and weighted classes of graphs) for which the proportional chromatic number can be calculated in polynomial time.

\section{References}

[1] J. Bibb Cain, T. Billhartz, L. Foore A link scheduling and ad hoc networking approach using directional antennas, Military comm. conf., 1 Oct 2003, 643-648.

[2] Carlson, R.C. and Nemhauser, G.L., Scheduling to minimize interaction cost, Oper. Res. 14 (1966), 52-58.

[3] Holyer, I. The NP-completeness of edge colourings, SIAM J. Computing 10 (1981), 718-720.

[4] Koreas, D.P., The NP-completeness of edge chromatic number in triangle free graphs with maximum vertex of degree 3, App. Math. and Comp. 83 (1) (1997), $13-17$.

[5] McDiarmid, C., Discrete mathematics and radio channel assignment, In: Recent advances in algorithms and combinatorics, eds. B. Reed and C. Linhares-Sales, Springer, New York, 2003, 27-63.

[6] G. L. Nemhauser, S. Park, A polyhedral approach to edge colouring, Operations Research Letters 10, 315-322, 1991.

[7] Vizing, V.G., On an estimate of a chromatic class of a p-graph (In Russian), Diskret. Analiz. 3 (1964), 25-30. 